\title{
Road Network Traffic Congestion Evaluation Simulation Model based on Complex Network
}

\author{
Chao Luo
}

Department of Information Management, Zhongnan University of Economics and Law, Wuhan , China

Key words: Traffic flow, Forecasting model, Congestion control, Simulation analysis

\begin{abstract}
To deeply analyze the dynamic evolution process of traffic congestion on road network and provide policy analysis tools for traffic congestion governance, the article proposed a traffic congestion evaluation simulation model on based on complex road network. By introducing section impedance concept into complex network theories, it achieved combination of topology model and traffic model on road network. Moreover, it proposed assessment methods on road network access capacity traversing in virtual test vehicles which could assess the road access capacity under different road network. At last, on PC system, it managed complete network congestion assessment simulation system, and made simulation analysis on factors affecting the access capacity such as number of road congestion, traffic flow and road network topology. The simulation results are same with classical theoretical analysis on traffic, and could reflect more dynamic process information, which shown that the model could make traffic network congestion assessment accurately and effectively as well as provide basis for related traffic congestion management decisions.
\end{abstract}

\section{Introduction}

In recent years, several researching groups had conducted researches on structural analysis of traffic network. Research results of Hui Yong Et al shown that public traffic network in Shanghai and Beijing presented a structural feature of Small World and Power Law. While Zong Yueguang research further proved the complex nature of network structure in traffic network. In his doctor papers, Wu Jianjun had made systematic analysis of complex network nature of traffic network structure, while Zhang Chen Et al.had analyzed the bus line Network Topology in Shanghai and Zhang Yong Et al. had conducted further study on robustness of traffic network on the basis of traffic network complexity. Traffic Flow Simulation had become a research focus in recent years, while the existing traffic simulation research still concentrated on simulation of dynamic evolution of microscopic traffic flow, which lack of consideration of traffic network structural factors. On the other hand, most of researches on traffic network concerned more about the structural characteristics of traffic network while lacked of traffic flow consideration. Through transmission dynamics characteristics of traffic, Li Shubin Et al. had studied the impact on traffic congestion by traffic network topology. Sun $\mathrm{H}$ Et al. had proposed the traffic dynamic theoretical model taking traffic network topology into consideration. By making full use of computing power of calculation simulation technology, the article would base on complex network theory and by section impedance model, introduce the traffic network congestion simulating evaluation taking traffic flow into consideration to achieve fine-grained.

\section{Construction of traffic network congestion evaluation and simulation model based on complex network}

\section{Complex network theory}

Small-World model was firstly proposed by D. Watts Et al. and was used to simulate relation of human and society. The following researches shown that many structures in human living environment could be presented by Small-World model, such as grid structure and traffic network. 
Small-World model could be described with Figure 1.

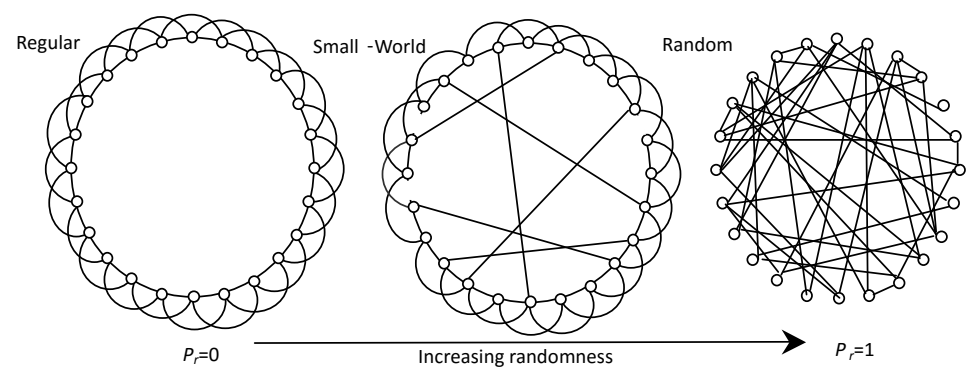

Figure 1 Small-World model of D. Watts

As shown in Figure 1, Small-World Topology was a topology between rule model (as shown in left side of Figure 1) and completely random model (as shown in right side of Figure 1). In Small-World structure, sides with some proportion (Pr) would be treated in random while most sides would remain a rule structure. Random sides connected knots with distant relation, thus it is also called "Short Cut". The mean value, which defined the minimum distance between two random points in the topology, is called characteristic path of the topology. The existence of random sides significantly shorten characteristic path length of the topology. Decrease of characteristic path promoted the accessibility and connectivity of topology. In following researches, several teams had studied the information transmission and robustness of Small-World structure [12].

Results of existing researches shown that there is Small-World structure in city traffic network. In certain area, sections had close connection while different areas were connected with main lines. Sections within area are equal to rule sides of Small-World while the main lines between areas could be regarded as Short Cut side of Small-World, subject to Small-World structure, a simulation model of traffic network could be created.

\section{Section impedance model}

Section impedance model was used to analyze the section delay issues caused by traffic flow, including time of vehicles through section and waiting time at intersection. Section impedance model was the most common model in practice as a computing model proposed by FHWA [2], as shown in Formula 1:

$$
t_{a}=t_{a}^{0}\left[1+\alpha\left({ }^{x} a / c_{a}^{\prime}\right)^{\beta}\right]
$$

Of which $t_{a}$ means section impedance, $t_{a}^{0}$ means the impedance value of section with traffic flow of $0 . \alpha$ and $\beta$ are correction factors, in general, $\alpha=0.15$ and $\beta=4.0 . x_{a}$ means flow on section and $c_{a}^{\prime}$ means the actual accessibility of section other than the maximum accessibility. When the flow increased to $c_{a}^{\prime}$, the related impedance value would be 1.15 times of $t_{a}$.

A problem lied in such model existence was that, when the traffic flow was infinitely close to section accessibility, the section impedance would not approach infinity, which is non-conformance to fact. In fact, when the traffic flow exceeded section accessibility, the section would be completed blocked, the infinity through section impedance would be regarded as the section was removed from the network. Thus, Davidson proposed a new section impedance calculation model, as shown in Formula 2[3]:

$$
t_{a}=t_{a}^{0}\left[1+J\left(^{x_{a}} /\left(c_{a}-x_{a}\right)\right]\right.
$$

Of which $\mathrm{J}$ is correction factor and $\mathrm{c}_{\mathrm{a}}$ is section accessibilty, $\mathrm{x}_{\mathrm{a}} / \mathrm{c}_{\mathrm{a}}$ is general unit in traffic engineering, usually called ratio of "flow/capacity" or congestion coefficient, other symbols in formula had the same meaning with that in Formula 1. According to Formula 2, when the congestion coefficient is 1 , the section impedance would approach infinity. When the traffic flow is 0 , the section impedance would be $t_{\mathrm{a}}^{0}$. Compared to the impedance model of FHWA, this model could better reflect the actual flow characteristics of the section. Thus, on construction of section 
impedance model, the article would choose this model to determine impedance in simulated traffic network.

It is obviously that the section impedance model could only consider the access conditions of single section other than the mutual impact of access conditions among different section in network. By combining the complex network model and section impedance model, the article constructed a traffic network congestion model which could take mutual of access conditions among different section in network, as a result to provide more effective supports to traffic management decisions.

\section{Analysis of simulation experiment}

By using Visual C++, the article achieved a complete simulation system on PC system and conducted simulation analysis by using the impact from the system on number of congestion sections, network traffic, flow changes on network accessibility and the impact from traffic network structure on network accessibility.

\section{Impact of number of congested sections on network accessibility}

Separately set network model as Power-Law structure with $\gamma=2.375$ and Small-World structure with $\mathrm{Pr}=0.01$, change the congested section proportion in network model to conduct simulation experiment. Congested section, mean a case that its flow $\mathrm{x}_{\mathrm{a}}$ reached to accessibility $\mathrm{c}_{\mathrm{a}}$ and the impedance $c_{a}$, approached infinity. The network size is of 10000 sections, single sections consisted $10 \%$. Except for congested sections, flow other sections is $10 \%$ of section accessibility, that means $c_{a}=1$ and $x_{a}=0.1$. The simulation results are as shown in Figure 2 as mean value after 100 simulations. Since the flow model was simplified, time was just a relative value in simulation experiment, it had no specific unit, however, such simplification would not affect the comparative analysis on network characteristics.

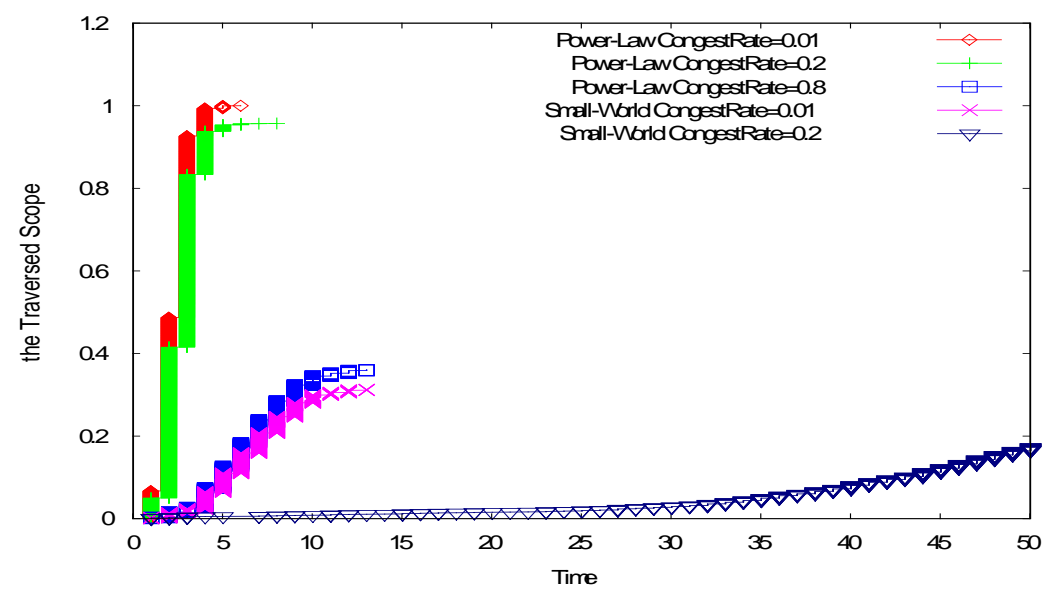

Figure 2 Impact of Number of Congested Sections on Network Accessibility

It could be inferred from Figure 2 that, as the number of congested sections increased, the network accessibility would gradually get worse. When the congested section proportion became only $10 \%$, there is almost no impact on accessibility of network model in Power-Law structure, however, when this proportion reached to $80 \%$, and took 14 time units, the virtual test vehicles would only traverse about $40 \%$ of the total network range, that means the network connectivity had been destroyed due to section congestion. Compared to Power-Law structure, the Small-World structure had worse resistance to network congestion, when there is $1 \%$ section congestion, network accessibility had been declined seriously, which is equal to $80 \%$ network congestion in Power-Law structure. In two structures, the knots number and sides representing sections are approximately equal. In case of $20 \%$ congestion, the traverse time in Small-World structure had reached to 45, while the traverse range is less than $20 \%$. In Small-World structure, the directional sides on knot connection had no big difference, but it had big difference in Power-Law structure, the most knots had about 100 directional sides, which differed from fact. But it could still be known that the 
Power-Law structure had stronger resistance to network congestion.

\section{Impact of flow changes on network accessibility}

In order to explore the impact of flow changes on network accessibility, we changed the section flow factor $\mathrm{x}_{\mathrm{a}}$ to conduct simulation experiment. Similar with the simulation experiment in 5.1, the experiment constructed the network model of Power-Law structure and Small-World structure at the same time, of which the Power-Law structure had $\gamma=2.375$ while the Small-World structure had $\operatorname{Pr}=0.01$, section number on network are both 10000 with $10 \%$ one-way sections. The congested sections are controlled at $1 \%$. Limited by length, the article would only consider the even distribution of flow in network model, the model could be expanded easily and could introduce OD flow model to simulate more true flow impacts. The results of simulation experiments are shown in Figure 3.
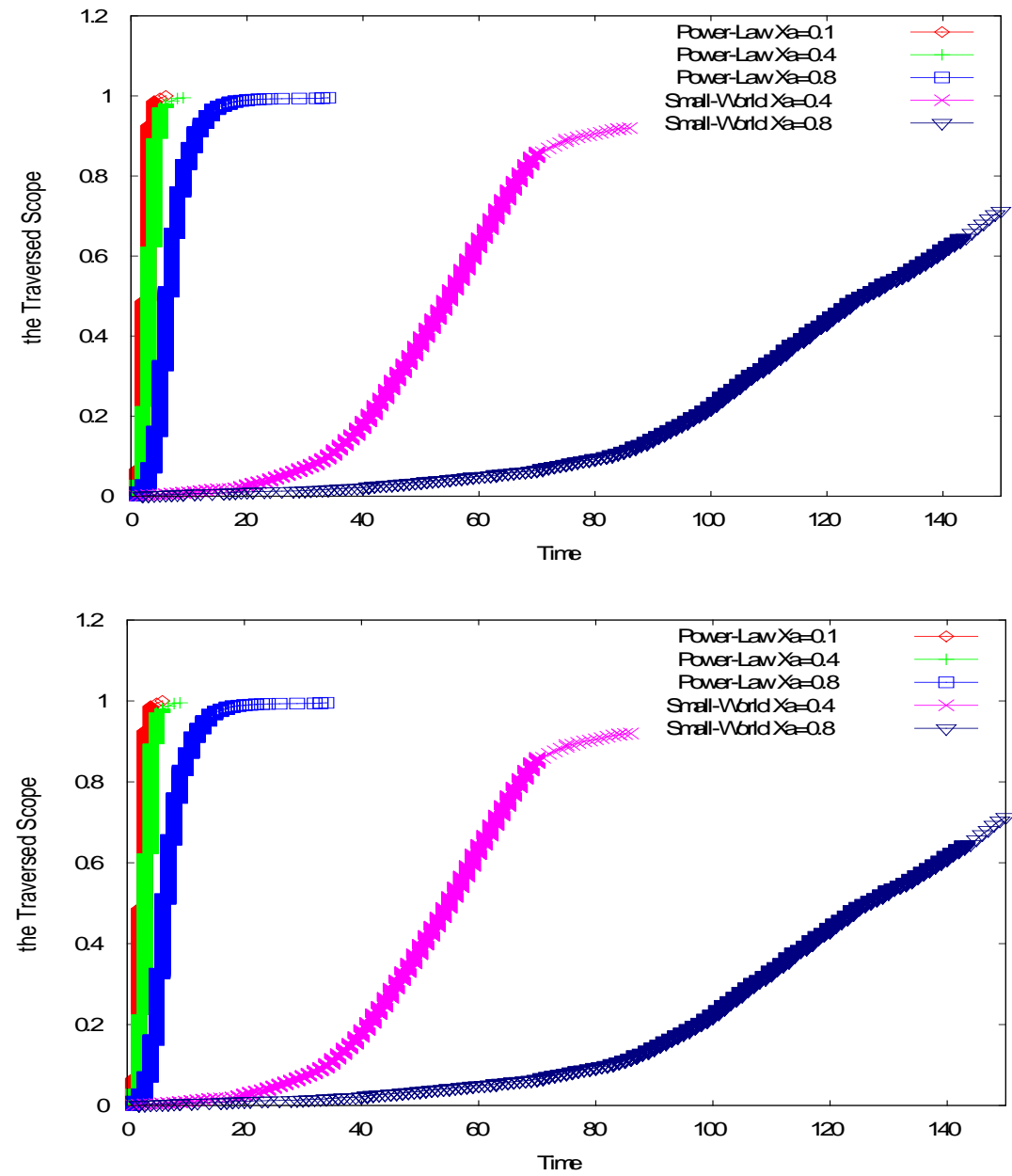

Figure 3 Impact of flow changes on network accessibility

It could be inferred from Figure 3 that, as flow increased, the network accessibility would decline, and the Small-World structure network is more sensitive to flow increases compared with Power-Law structure network. When $\mathrm{x}_{\mathrm{a}}=0.4,90 \%$ traverse and more of Power-Law structure network would take 40 time units, while under the same flow circumstance, Small-World structure network would take 100. And as flow increased, such difference would continue to enlarge, when the flow increased to $80 \%$ of network accessibility, the traverse time of Small-World structure network would increase in sharp, and the network would be at the edge of complete congestion. 


\section{Network structure changes on network accessibility}

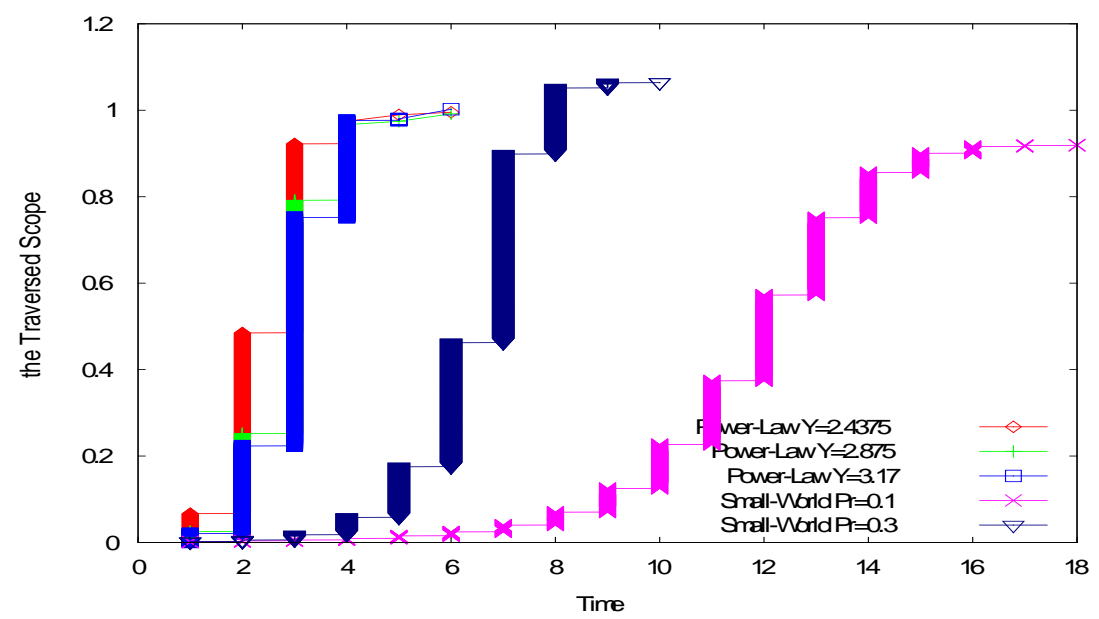

Figure 4 Network Topology Impact on Network Accessibility

According to complex network theory, with different network structure parameters, the topology characteristics would change accordingly. We could see from previous experiments that, network topology had greater impact on network accessibility. In order to conduct further study on network topology impact on network accessibility, the article would change the network structure parameter to conduction simulation experiment. In this experiment, we assume congested section proportion as $1 \%$ and traffic flow had reached $1 \%$ of network accessibility, rest parameters remained the same with previous experiments. The results of simulation experiments are shown in Figure 4.

It could be inferred from Figure 4 that the network topology characteristic changes had some impact on network accessibility. For Power-Law structure, as $\gamma$ increased, the network accessibility would present a certain decline. With $\gamma$ increasing, the knots with less out-degree in network would increase proportion of occupied sections, which means the section would be distributed more evenly. Thus, in Power-Law structure, it is those knots with large out-degree could affect the accessibility. While in Small-World structure, as Pr increased, network accessibility would enlarge significantly. When $\mathrm{Pr}=0.3$, the accessibility of Small-World structure would approach to that of Power-Law structure, and the number of "Short Cut" sides would also increase. This shows that for Small-World structure, it is "Short Cut" sides which could affect the accessibility. While without adding network section numbers and adding access among different central areas as much as possible would be an effective way to promote the network accessibility.

\section{Conclusions}

On the basis of complex network theory and taking use of Monte Carlo simulation technology, the article constructed network traffic congestion simulation evaluation model. The model combined the complex network theory and traffic flow theory by introducing section impedance concept, thus it could analyze the mutual impact on relation among traffic congestion on different sections in network system in quantitative, and provide supports to traffic flow management and transportation management optimization. Limited by length, the article had simplified the traffic flow model, provided that the model met the necessary simulation fidelity. In following works, the article would, on the basis of OD Matrix Estimation Theory, further improve the simulation model, to make it could conduct simulation of network traffic congestion dynamic evolution with finer-grained and provide decision basis for traffic congestion control.

\section{Reference}

[1] Ying Liang, Yingying Yi, Qiufen Sun. (2014). The Impact of Migration on Fertility under China's Underlying Restrictions: A Comparative Study Between Permanent and Temporary Migrants, Social Indicators Research, 116(1): 307-326. 
[2] Liu Y, Yang J, Meng Q, et al. Stereoscopic image quality assessment method based on binocular combination saliency model[J]. Signal Processing, 2016, 125: 237-248.

[3] Li Su, Hanwei Wang, Jingwei Miao ,Ying Liang.(2015).Clinicopathological Significance and Potential Drug Target of CDKN(2)A/p(16) in Endometrial Carcinoma, Scientific Reports, 5:13238, 2015. 1-7.

[4] Jiang D, Xu Z, Lv Z. A multicast delivery approach with minimum energy consumption for wireless multi-hop networks[J]. Telecommunication Systems, 2015: 1-12.

[5] Lv, Z., Chirivella, J., \& Gagliardo, P. (2016). Bigdata Oriented Multimedia Mobile Health Applications. Journal of medical systems, 40(5), 1-10. 